\title{
Some Coupled fixed point theorems in partially ordered $A_{b}$-metric space
}

\author{
N. Mlaiki ${ }^{\mathrm{a}, *}$, Y. Rohen ${ }^{\mathrm{b}}$ \\ ${ }^{a}$ Department of Mathematical Sciences, Prince Sultan University, Riyadh, Saudi Arabia. \\ ${ }^{b}$ Department of Mathematics, NIT Manipur, Langol, Imphal, India.
}

Communicated by F. Vetro

\begin{abstract}
In this paper, we use the concept of $A_{b}$-metric space which is obtained by generalizing the definitions of $A$-metric space and $b$-metrc space. Using this concept, we prove some coupled fixed point theorems in partially ordered $A_{b}$-metric space. Examples are also presented to verify the obtained results. (C)2017 All rights reserved.
\end{abstract}

Keywords: Common fixed points, A-metric space, b-metric space, $A_{b}$-metric space. 2010 MSC: 54H25, 47H10.

\section{Introduction and preliminaries}

In the study of fixed point, the generalization of metric space is one of the interesting topics for many researchers. Some of the generalizations of metric space are 2-metric space, D-metric space, $\mathrm{D}^{*}$-metric space, G-metric space, S-metric space, rectangular metric or metric-like space, partial metric space, fuzzy metric space, probabilistic metric space, cone metric space, modular metric space, etc.

In the year 1989, Bhaktin [5] introduced the concept of b-metric space in which the triangle inequality is made by a more general form by introducing a scale factor which is a real number greater than or equal to one. By doing so the ordinary metric space becomes a special case of $b$-metric space when scale factor is equal to one.

Introduction of b-metric space opens a gate to further generalization of metric space. Due to the introduction of $b$-metric space, many generalizations of metric space came into existence. Mention can be made about $G_{b}$-metric space, $S_{b}$-metric space, $b_{2}$-metric space, cone b-metric space, partial b-metric space, fuzzy b-metric space, rectangular b-metric space etc. Some results about generalized metric space can be seen in $[1,5,8,12,15-19,21-23]$ and references there in.

Recently, Abbas et al. [1] introduced the concept of an n-tuple metric space and studied its topological properties. This new structure is named as A-metric space. The definition of A-metric is as follows.

Definition 1.1 ([1]). Let $X$ be a nonempty set. A function $A: X^{n} \rightarrow[0, \infty)$ is called an $A$-metric on $X$ if for any $x_{i}, a \in X, i=1,2, \ldots, n$, the following conditions hold:

\footnotetext{
*Corresponding author

Email addresses: nmlaiki@psu.edu.sa (N. Mlaiki), ymnehor2008@yahoo.com (Y. Rohen)
} 
(A1) $A\left(x_{1}, x_{2}, x_{3}, \ldots, x_{n-1}, x_{n}\right) \geqslant 0$,

(A2) $A\left(x_{1}, x_{2}, x_{3}, \ldots, x_{n-1}, x_{n}\right)=0$ if and only if $x_{1}=x_{2}=x_{3}=\ldots=x_{n-1}=x_{n}$,

(A3)

$$
\begin{aligned}
A\left(x_{1}, x_{2}, x_{3}, \ldots, x_{n-1}, x_{n}\right) \leqslant & {\left[A\left(x_{1}, x_{1}, x_{1}, \ldots,\left(x_{1}\right)_{n-1}, a\right)\right.} \\
& +A\left(x_{2}, x_{2}, x_{2}, \ldots,\left(x_{2}\right)_{n-1}, a\right) \\
& +A\left(x_{3}, x_{3}, x_{3}, \ldots,\left(x_{3}\right)_{n-1}, a\right) \\
& \vdots \\
& +A\left(x_{n-1}, x_{n-1}, x_{n-1}, \ldots,\left(x_{n-1}\right)_{n-1}, a\right) \\
& \left.+A\left(x_{n}, x_{n}, x_{n}, \ldots,\left(x_{n}\right)_{n-1}, a\right)\right] .
\end{aligned}
$$

The pair $(X, A)$ is called an $A$-metric space.

Some examples of A-metric space are given in [1].

Example 1.2 ([1]). Let $X=[1,+\infty)$. Define $A: X^{n} \rightarrow[0,+\infty)$ by

$$
A\left(x_{1}, x_{2}, x_{3}, \ldots, x_{n-1}, x_{n}\right)=\sum_{i=1}^{n} \sum_{i<j}\left|x_{i}-x_{j}\right|
$$

for all $x_{i} \in X, i=1,2, \ldots n$. Then $(X, A)$ is an $A$-metric space.

Example $1.3([1])$. Let $X=\mathbb{R}$. Define $A: X^{n} \rightarrow[1,+\infty)$ by

$$
\begin{aligned}
A_{b}\left(x_{1}, x_{2}, x_{3}, \ldots, x_{n-1}, x_{n}\right)= & \left|\sum_{i=2}^{n} x_{i}-(n-1) x_{1}\right|+\left|\sum_{i=3}^{n} x_{i}-(n-2) x_{2}\right|+\cdots \\
& +\left|\sum_{i=n-3}^{n} x_{i}-3 x_{n-3}\right|+\left|\sum_{i=n-2}^{n} x_{i}-2 x_{n-2}\right|+\left|x_{n}-x_{n-1}\right|
\end{aligned}
$$

for all $x_{i} \in X, i=1,2, \ldots n$. Then $(X, A)$ is an A-metric space.

The concept of b-metric space was introduced by Bakhtin [5]. The definition of b-metric space is as follows.

Definition 1.4 ([6]). Let $X$ be a nonempty set. A b-metric on $X$ is a function $d: X^{2} \rightarrow[0, \infty)$ if there exists a real number $s \geqslant 1$ such that the following conditions hold for all $x, y, z \in X$ :

(i) $d(x, y)=0$ if and only if $x=y$;

(ii) $d(x, y)=d(y, x)$;

(iii) $d(x, z) \leqslant s[d(x, y)+d(y, z)]$.

The pair $(X, d)$ is called a b-metric space.

Motivated by the concepts given by Abbas et al. [1] and Bakhtin [5], Ughade et al. [23] introduced a generalized form of $n$-tuple metric space. They named it as $A_{b}$-metric space and defined as follows.

Definition 1.5 ([23]). Let $X$ be a nonempty set and $b \geqslant 1$ be a given real number. A function $A: X^{n} \rightarrow$ $[0, \infty)$ is called an $A_{b}$-metric on $X$ if for any $x_{i}, a \in X, i=1,2, \ldots, n$, the following conditions hold:

$\left(A_{b} 1\right) A\left(x_{1}, x_{2}, x_{3}, \ldots, x_{n-1}, x_{n}\right) \geqslant 0$;

$\left(A_{b} 2\right) A\left(x_{1}, x_{2}, x_{3}, \ldots, x_{n-1}, x_{n}\right)=0$ if and only if $x_{1}=x_{2}=x_{3}=\ldots=x_{n-1}=x_{n}$; 
$\left(A_{b} 3\right)$

$$
\begin{aligned}
A\left(x_{1}, x_{2}, x_{3}, \ldots, x_{n-1}, x_{n}\right) \leqslant & b\left[A\left(x_{1}, x_{1}, x_{1}, \ldots,\left(x_{1}\right)_{n-1}, a\right)\right. \\
& +A\left(x_{2}, x_{2}, x_{2}, \ldots,\left(x_{2}\right)_{n-1}, a\right) \\
& +A\left(x_{3}, x_{3}, x_{3}, \ldots,\left(x_{3}\right)_{n-1}, a\right) \\
& \vdots \\
& +A\left(x_{n-1}, x_{n-1}, x_{n-1}, \ldots,\left(x_{n-1}\right)_{n-1}, a\right) \\
& \left.+A\left(x_{n}, x_{n}, x_{n}, \ldots,\left(x_{n}\right)_{n-1}, a\right)\right] .
\end{aligned}
$$

The pair $\left(X, A_{b}\right)$ is called an $A_{b}$-metric space.

Definition 1.6. An $A_{b}$-metric space is said to be symmetric if $A\left(x_{1}, x_{1}, \ldots,\left(x_{1}\right)_{n-1}, x_{2}\right)=A\left(x_{2}, x_{2}, \ldots\right.$, $\left.\left(x_{2}\right)_{\mathfrak{n}-1}, x_{1}\right)$.

Note: $A_{b}$-metric space is more general than A-metric space. Moreover A-metric space is a special case of $A_{b}$-metric space with $b=1$. $b$-metric space and $S_{b}$-metric space are also special cases of $A_{b}$-metric space with $n=2$ and 3 , respectively. Ordinary metric space and $S$-metric space are also special cases of $A_{b}$-metric space with $b=1$ and respective values of $n$ as 2 and 3 .

Example $1.7([23])$. Let $X=[1,+\infty)$. Define $A_{b}: X^{n} \rightarrow[1,+\infty)$ by

$$
A_{b}\left(x_{1}, x_{2}, x_{3}, \ldots, x_{n-1}, x_{n}\right)=\sum_{i=1}^{n} \sum_{i<j}\left|x_{i}-x_{j}\right|^{2}
$$

for all $x_{i} \in X, i=1,2, \ldots n$. Then $\left(X, A_{b}\right)$ is an $A_{b}$-metric space with $s=2>1$.

Example 1.8 ([23]). Let $X=\mathbb{R}$. Define $A_{b}: X^{n} \rightarrow[1,+\infty)$ by

$$
\begin{aligned}
A_{b}\left(x_{1}, x_{2}, x_{3}, \ldots, x_{n-1}, x_{n}\right)= & \left|\sum_{i=2}^{n} x_{i}-(n-1) x_{1}\right|^{2}+\left|\sum_{i=3}^{n} x_{i}-(n-2) x_{2}\right|^{2}+\cdots \\
& +\left|\sum_{i=n-3}^{n} x_{i}-3 x_{n-3}\right|^{2}+\left|\sum_{i=n-2}^{n} x_{i}-2 x_{n-2}\right|^{2}+\left|x_{n}-x_{n-1}\right|^{2}
\end{aligned}
$$

for all $x_{i} \in X, i=1,2, \ldots n$. Then $\left(X, A_{b}\right)$ is an $A_{b}$-metric space with $s=2>1$

Example 1.9. Let $X=[1, \infty)$. Define a function $A_{b}: X^{n} \rightarrow[0, \infty)$ by $A_{b}\left(x_{1}, x_{2}, \cdots, x_{n}\right)=\mid x_{1}-$ $\left.\max \left\{x_{2}, \cdots, x_{n}\right\}\right|^{2}$ for all $x_{1}, x_{2}, \cdots, x_{n} \in X$. Then $\left(X, A_{b}\right)$ is an $A_{b}$-metric on $X$ with $b=2$, and it is not difficult to see that $\left(X, A_{b}\right)$ is not an $A$-metric space on $X$.

Lemma 1.10 ([23]). Let $(X, A)$ be $A_{b}$-metric space. Then $A(x, x, x, \ldots, x, y) \leqslant b A(y, y, y, \ldots, y, x)$ for all $x, y \in X$.

Lemma 1.11. Let $(X, A)$ be $A_{b}$-metric space. Then for all $x, y, z \in X$ we have $A(x, x, x, \ldots, x, z) \leqslant(n-$ 1) $b A(x, x, x, \ldots, x, y)+b^{2} A(y, y, y, \ldots, y, z)$.

Proof. By $\left(A_{\mathrm{b}} 3\right)$, we have

$$
\begin{aligned}
A(x, x, x, \ldots, x, y) & \leqslant b[A(x, x, x, \ldots, x, y)+A(x, x, x, \ldots, x, y)+\cdots+(n-1) \text { times }+A(z, z, z, \ldots, z, y)] \\
& \leqslant b[(n-1) A(x, x, x, \ldots, x, y)+b A(y, y, y, \ldots, y, z)] \\
& =(n-1) b A(x, x, x, \ldots, x, y)+b^{2} A(y, y, y, \ldots, y, z) .
\end{aligned}
$$

Lemma 1.12. Let $(X, A)$ be $A_{b}$-metric space. Then $\left(X^{2}, D_{A}\right)$ is $A_{b}$-metric space on $X \times X$ with the metric $D_{A}$ given by $\mathrm{D}\left(\left(x_{1}, y_{1}\right),\left(x_{2}, y_{2}\right), \ldots,\left(x_{n}, y_{n}\right)\right)=A\left(x_{1}, x_{1}, x_{1}, \ldots, x_{n}\right)+A\left(y_{1}, y_{2}, y_{3}, \ldots, y_{n}\right)$ for all $x_{i}, y_{j} \in X, i, j=$ $1,2, \ldots, n$. 
Definition 1.13. The $A_{b}$-metric space $(X, A)$ is said to be bounded if there exists a constant $r>0$ such that $A(x, x, x, \ldots, x, y) \leqslant r$ for all $x, y \in X$. Otherwise, $X$ is unbounded.

Definition 1.14. Given a point $x_{0}$ in $A_{b}$-metric space $(X, A)$ and a positive real number $r$, the set $B\left(x_{0}, r\right)=$ $\left\{y \in X: A\left(y, y, y, \ldots, y, x_{0}\right)<r\right\}$ is called an open ball centered at $x_{0}$ with radius $r$.

The set $\overline{B\left(x_{0}, r\right)}=\left\{y \in X: A\left(y, y, y, \ldots, y, x_{0}\right) \leqslant r\right\}$ is called a closed ball centered at $x_{0}$ with radius $r$.

Definition 1.15. A subset $G$ in $A_{b}$-metric space $(X, A)$ is said to be an open set if for each $x \in G$ there exists an $r>0$ such that $B(x, r) \subset G$. A subset $F \subset X$ is called closed if $X \backslash F$ is open.

Lemma 1.16. In any $\mathrm{A}_{\mathrm{b}}$-metric space $(\mathrm{X}, \mathrm{A})$, each open ball is an open set in $\mathrm{X}$ and each closed ball is also a closed set in $\mathrm{X}$.

Theorem 1.17. Let $(X, A)$ be $A_{b}$-metric space, then:

(i) An arbitrary union and finite intersection of open balls $\mathrm{B}(\mathrm{x}, \mathrm{r}) \in \mathrm{X}$ is open.

(ii) An arbitrary intersection and finite union of closed balls $\mathrm{B}(\mathrm{x}, \mathrm{r}) \in \mathrm{X}$ is closed.

Theorem 1.18. The collection $T=\{B(x, r): x \in X, r>0\}$ of all balls in $A_{b}$-metric space $(X, A)$ is a basis for a topology $\tau$ on $\mathrm{X}$.

Definition 1.19. Let $(X, A)$ be $A_{b}$-metric space. A sequence $\left\{x_{k}\right\}$ in $X$ is said to converge to a point $x \in X$, if $A\left(x_{k}, x_{k}, x_{k}, \ldots, x_{k}, x\right) \rightarrow 0$ as $k \rightarrow \infty$. That is, for each $\varepsilon \geqslant 0$, there exists $N \in \mathbb{N}$ such that for all $k \geqslant N$ we have $A\left(x_{k}, x_{k}, x_{k}, \ldots, x_{k}, x\right) \leqslant \varepsilon$ and we write $\lim _{k \rightarrow \infty} x_{k}=x$.

Lemma 1.20. Let $(X, A)$ be $A_{b}$-metric space. If the sequence $\left\{x_{k}\right\}$ in $X$ converges to a point $x$, then $x$ is unique.

Proof. Suppose $\left\{x_{k}\right\}$ converges to $x$ and $y$. Then, for $\varepsilon>0$, there exists $N_{1}, N_{2} \in \mathbb{N}$ such that for all $k \geqslant N_{1}$ we have $A\left(x_{k}, x_{k}, \ldots, x_{k}, x\right)<\frac{\varepsilon}{2 b^{2}(n-1)}$ and for every $k \geqslant N_{2}$, we get $A\left(x_{k}, x_{k}, \ldots, x_{k}, y\right)<\frac{\varepsilon}{2 b^{2}}$. Choose $\mathrm{N}=\max \left\{\mathrm{N}_{1}, \mathrm{~N}_{2}\right\}$, therefore, for all $k \geqslant N$, we have

$$
\begin{aligned}
A(x, x, \ldots, x, y) & =(n-1) b A\left(x, x, \ldots, x, x_{k}\right)+b^{2} A\left(x_{k}, x_{k}, \ldots, x_{k}, y\right) \\
& \leqslant(n-1) b^{2} A\left(x_{k}, x_{k}, \ldots, x_{k}, x\right)+b^{2} A\left(x_{k}, x_{k}, \ldots, x_{k}, y\right) \\
& \leqslant(n-1) b^{2} \frac{\varepsilon}{2 b^{2}(n-1)}+b^{2} \times \frac{\varepsilon}{2 b^{2}} \\
& =\varepsilon .
\end{aligned}
$$

Since $\varepsilon$ is arbitrary, we have $A(x, x, \ldots, x, y)=0$ and so $x=y$. Establishing the uniqueness of $\left\{x_{k}\right\}$.

Definition 1.21. Let $(X, A)$ be $A_{b}$-metric space. A sequence $\left\{x_{k}\right\}$ in $X$ is called a Cauchy sequence if $A\left(x_{k}, x_{k}, x_{k}, \ldots, x_{k}, x_{m}\right) \rightarrow 0$ as $k, m \rightarrow \infty$.

That is, for each $\epsilon \geqslant 0$, there exists $N \in \mathbb{N}$ such that for all $k, m \in \mathbb{N}$ we have $A\left(x_{k}, x_{k}, x_{k}, \ldots, x_{k}, x_{m}\right) \leqslant$ $\epsilon$ and we write $\lim _{k \rightarrow \infty} x_{k}=x$.

Lemma 1.22. Every convergent sequence in $A_{b}$-metric space is a Cauchy sequence.

Proof. Let $\left\{x_{k}\right\}$ be a convergent sequence in $(X, A)$. Let $\lim _{k \rightarrow \infty} x_{k}=x$. Then, given $\varepsilon>0$, there exists $N_{1}, N_{2} \in \mathbb{N}$ such that for all $k \geqslant N_{1}$ we have $A\left(x_{k}, x_{k}, \ldots, x_{k}, x\right)<\frac{\varepsilon}{2 b(n-1)}$ and for every $m \geqslant N_{2}$, we get $A\left(x_{m}, x_{m}, \ldots, x_{m}, x\right)<\frac{\varepsilon}{2 b^{2}}$. Putting $N=\max \left\{N_{1}, N_{2}\right\}$. Therefore, for all $k, m \geqslant N$, we obtain

$$
\begin{aligned}
A\left(x_{k}, x_{k}, \ldots, x_{k}, x_{m}\right) & =(n-1) b A\left(x_{k}, x_{k}, \ldots, x_{k}, x\right)+b^{2} A\left(x_{m}, x_{m}, \ldots, x_{m}, x\right) \\
& \leqslant(n-1) b \frac{\varepsilon}{2 b(n-1)}+b^{2} \times \frac{\varepsilon}{2 b^{2}} \\
& =\varepsilon .
\end{aligned}
$$

This implies that $\left\{x_{n}\right\}$ is a Cauchy sequence. 
Remark 1.23. The converse of Lemma 1.22 does not hold in general. A Cauchy sequence in an $A_{b}$-metric space does not need to be convergent.

Definition 1.24. The $A_{b}$-metric space $(X, A)$ is said to be complete if every Cauchy sequence in $X$ is convergent.

Definition 1.25. Let $\left(X, A_{X}\right)$ and $\left(Z, A_{Z}\right)$ be $A_{b}$-metric spaces. A function $f: X \rightarrow Z$ is continuous at a point $x_{0} \in X$, if $f^{-1}(G)$ is open in $X$ for each open set $G$ in $Z$. The function $f$ is continuous on $X$ if it is continuous at each points of $X$.

Theorem 1.26. Let $\left(X, A_{X}\right)$ and $\left(Z, A_{Z}\right)$ be $A_{b}$-metric spaces. A function $f: X \rightarrow Z$ is continuous at a point $\mathrm{x}_{0} \in \mathrm{X}$ iff it is sequentially continuous at $\mathrm{x}_{0}$.

Lemma 1.27. Let $(X, A)$ be $A_{b}$-metric space, then the function $A(x, x, x, \ldots, x, y)$ is continuous in all of its arguments. In other words, if there exist sequences $\left\{x_{k}\right\}$ and $\left\{y_{k}\right\}$ such that $\lim _{k \rightarrow \infty} x_{k}=x$ and $\lim _{k \rightarrow \infty} y_{k}=y$, then $\lim _{k \rightarrow \infty} A\left(x_{k}, x_{k}, x_{k}, \ldots, x_{k}, y_{k}\right)=A(x, x, x, \ldots, x, y)$.

Definition 1.28. An element $(x, y) \in X \times X$ is called a coupled fixed point of the mapping $S: X \times X \rightarrow X$ if

$$
S(x, y)=x, \text { and } S(y, x)=y .
$$

In 1987 Guo and Lakshmikantham [7] introduced the concept of coupled fixed point. Later, Bhaskar and Lakshmikantham [6] proved a new fixed point theorem for a mixed monotone mapping in a metric space powered with partial ordered by using a weak contractivity type assumption. For more results on coupled fixed point results one can see research results in $[2-5,7,9-11,13,14,20]$ and references therein.

\section{Main result}

In this section, we obtain common coupled fixed point results of mappings satisfying more general contractive conditions in the framework of partially ordered $A_{b}$-metric spaces. We start with the following result.

Theorem 2.1. Let $(X, \preceq, A)$ be a partially ordered complete $A_{b}$-metric space, and $f, g: X^{2} \rightarrow X$ be two maps such that

1. the pair $(\mathrm{f}, \mathrm{g})$ has mixed weakly monotone property on $\mathrm{X}$,

$$
x_{0} \preceq f\left(x_{0}, y_{0}\right), f\left(y_{0}, x_{0}\right) \preceq y_{0} \text { or } x_{0} \preceq g\left(x_{0}, y_{0}\right), g\left(y_{0}, x_{0}\right) \leqslant y_{0} \text { for some } x_{0}, y_{0} \in X ;
$$

2. there exist $a_{i} \succeq 0, i=1, \ldots, 5$, satisfying $a_{1}+a_{2}+b^{2}\left(a_{3}+a_{4}\right)+a_{5}<1$ and

$$
\begin{aligned}
& A(f(x, y), f(x, y), \ldots, f(x, y), g(u, v))+A(f(y, x), f(y, x), \ldots, f(y, x), g(v, u)) \\
& \leqslant \\
& \quad a_{1} D((x, y),(x, y), \ldots,(x, y),(u, v))+a_{2} D((x, y),(x, y), \ldots,(x, y),(f(x, y), f(y, x))) \\
& \quad+a_{3} D((u, v),(u, v), \ldots,(u, v),(g(u, v), g(v, u))) \\
& \quad+a_{4} D((x, y),(x, y), \ldots,(x, y),(g(u, v), g(v, u))) \\
& \quad+a_{5} D((u, v),(u, v), \ldots,(u, v),(f(x, y), f(y, x)))
\end{aligned}
$$

for all $x, y, u, v \in X$ with $x \preceq u$ and $y \succeq v$;

3. either $f$ or $g$ is continuous or $X$ has the following properties:

(a) if $\left\{x_{k}\right\}$ is an increasing sequence with $x_{k} \rightarrow x$, then $x_{k} \preceq x$ for all $k \in \mathbb{N}$;

(b) if $\left\{y_{k}\right\}$ is a decreasing sequence with $y_{k} \rightarrow y$, then $y \preceq y_{k}$ for all $k \in \mathbb{N}$.

Then $\mathrm{f}$ and $\mathrm{g}$ have a coupled common fixed point in $\mathrm{X}$. 
Proof. Let $\left(x_{0}, y_{0}\right)$ be a given point in $X \times X$. Choose $x_{1}=f\left(x_{0}, y_{0}\right), y_{1}=f\left(y_{0}, x_{0}\right), x_{2}=g\left(x_{1}, y_{1}\right)$ and $y_{2}=g\left(y_{1}, x_{1}\right)$. From the condition $x_{0} \preceq f\left(x_{0}, y_{0}\right), y_{0} \succeq f\left(y_{0}, x_{0}\right)$, and the fact that $(f, g)$ has mixed weakly monotone property we have

$$
x_{1}=f\left(x_{0}, y_{0}\right) \preceq g\left(f\left(x_{0}, y_{0}\right), f\left(y_{0}, x_{0}\right)\right)=g\left(x_{1}, y_{1}\right) \Rightarrow x_{1} \preceq x_{2}
$$

and

$$
x_{2}=g\left(x_{1}, y_{1}\right) \preceq f\left(g\left(x_{1}, y_{1}\right), g\left(y_{1}, x_{1}\right)\right)=f\left(x_{2}, y_{2}\right) \Rightarrow x_{2} \preceq x_{3} .
$$

Thus,

and

$$
y_{1}=f\left(x_{0}, y_{0}\right) \succeq g\left(f\left(y_{0}, x_{0}\right), f\left(x_{0}, y_{0}\right)\right)=g\left(y_{1}, x_{1}\right) \Rightarrow y_{1} \succeq y_{2}
$$

$$
y_{2}=g\left(y_{1}, x_{1}\right) \succeq f\left(g\left(y_{1}, x_{1}\right), g\left(x_{1}, y_{1}\right)\right)=f\left(y_{2}, x_{2}\right) \Rightarrow y_{2} \succeq y_{3} .
$$

Continuing this way, we obtain

$$
\begin{aligned}
& x_{2 k+1}=f\left(x_{2 k}, y_{2 k}\right), y_{2 k+1}=f\left(y_{2 k}, x_{2 k}\right), \\
& x_{2 k+2}=g\left(x_{2 k+1}, y_{2 k+1}\right), y_{2 k+2}=g\left(y_{2 k+1}, x_{2 k+1}\right) \text { for all } k \in \mathbb{N} .
\end{aligned}
$$

Therefore the sequences $\left\{x_{k}\right\}$ and $\left\{y_{k}\right\}$ are monotone:

$$
\begin{aligned}
& x_{0} \preceq x_{1} \preceq x_{2} \preceq x_{3} \preceq \cdots \preceq x_{k} \preceq x_{2 k+1} \preceq x_{2 k+2} \preceq \cdots, \\
& y_{0} \succeq y_{1} \succeq y_{2} \succeq y_{3} \succeq \cdots \succeq y_{k} \succeq y_{2 k+1} \succeq y_{2 k+2} \succeq \cdots
\end{aligned}
$$

Now we show that these sequences are Cauchy. From the contractive condition (2.1) we have for all $k \in \mathbb{N}$

$$
\begin{aligned}
& A\left(x_{2 k+1}, x_{2 k+1}, \ldots, x_{2 k+1}, x_{2 k+2}\right)+A\left(y_{2 k+1}, y_{2 k+1}, \ldots, y_{2 k+1}, y_{2 k+2}\right) \\
&= A\left(f\left(x_{2 k}, y_{2 k}\right), f\left(x_{2 k}, y_{2 k}\right), \ldots, f\left(x_{2 k}, y_{2 k}\right), g\left(x_{2 k+1}, y_{2 k+1}\right)\right) \\
&+A\left(f\left(y_{2 k}, x_{2 k}\right), f\left(y_{2 k}, x_{2 k}\right), \ldots, f\left(y_{2 k}, x_{2 k}\right), g\left(y_{2 k+1}, x_{2 k+1}\right)\right) \\
& \leqslant a_{1} D\left(\left(x_{2 k}, y_{2 k}\right),\left(x_{2 k}, y_{2 k}\right), \ldots,\left(x_{2 k}, y_{2 k}\right),\left(x_{2 k+1}, y_{2 k+1}\right)\right) \\
&+a_{2} D\left(\left(x_{2 k}, y_{2 k}\right),\left(x_{2 k}, y_{2 k}\right), \ldots,\left(x_{2 k}, y_{2 k}\right),\left(f\left(x_{2 k}, y_{2 k}\right), f\left(y_{2 k}, x_{2 k}\right)\right)\right) \\
&+a_{3} D\left(\left(x_{2 k+1}, y_{2 k+1}\right),\left(x_{2 k+1}, y_{2 k+1}\right), \ldots,\left(x_{2 k+1}, y_{2 k+1}\right),\left(g\left(x_{2 k+1}, y_{2 k+1}\right), g\left(y_{2 k+1}, x_{2 k+1}\right)\right)\right) \\
&+a_{4} D\left(\left(x_{2 k}, y_{2 k}\right),\left(x_{2 k}, y_{2 k}\right), \ldots,\left(x_{2 k}, y_{2 k}\right),\left(g\left(x_{2 k+1}, y_{2 k+1}\right), g\left(y_{2 k+1}, x_{2 k+1}\right)\right)\right) \\
&+a_{5} D\left(\left(x_{2 k+1}, y_{2 k+1}\right),\left(x_{2 k+1}, y_{2 k+1}\right), \ldots,\left(x_{2 k+1}, y_{2 k+1}\right),\left(f\left(x_{2 k}, y_{2 k}\right), f\left(y_{2 k}, x_{2 k}\right)\right)\right) .
\end{aligned}
$$

Applying (2.2), we get

$$
\begin{aligned}
A\left(x_{2 k}+1\right. & \left.x_{2 k+1}, \ldots, x_{2 k+1}, x_{2 k+2}\right)+A\left(y_{2 k+1}, y_{2 k+1}, \ldots, y_{2 k+1}, y_{2 k+2}\right) \\
\leqslant & a_{1} D\left(\left(x_{2 k}, y_{2 k}\right),\left(x_{2 k}, y_{2 k}\right), \ldots,\left(x_{2 k}, y_{2 k}\right),\left(x_{2 k+1}, y_{2 k+1}\right)\right) \\
& +a_{2} D\left(\left(x_{2 k}, y_{2 k}\right),\left(x_{2 k}, y_{2 k}\right), \ldots,\left(x_{2 k}, y_{2 k}\right),\left(x_{2 k+1}, y_{2 k+1}\right)\right) \\
& +a_{3} D\left(\left(x_{2 k+1}, y_{2 k+1}\right),\left(x_{2 k+1}, y_{2 k+1}\right), \ldots,\left(x_{2 k+1}, y_{2 k+1}\right),\left(x_{2 k+2}, y_{2 k+2}\right)\right) \\
& +a_{4} D\left(\left(x_{2 k}, y_{2 k}\right),\left(x_{2 k}, y_{2 k}\right), \ldots,\left(x_{2 k}, y_{2 k}\right),\left(x_{2 k+2}, y_{2 k+2}\right)\right) \\
& +a_{5} D\left(\left(x_{2 k+1}, y_{2 k+1}\right),\left(x_{2 k+1}, y_{2 k+1}\right), \ldots,\left(x_{2 k+1}, y_{2 k+1}\right),\left(x_{2 k+1}, y_{2 k+1}\right)\right) \\
= & a_{1} D\left(\left(x_{2 k}, y_{2 k}\right),\left(x_{2 k}, y_{2 k}\right), \ldots,\left(x_{2 k}, y_{2 k}\right),\left(x_{2 k+1}, y_{2 k+1}\right)\right) \\
& \left.+a_{2} D\left(\left(x_{2 k}, y_{2 k}\right),\left(x_{2 k}, y_{2 k}\right), \ldots,\left(x_{2 k}, y_{2 k}\right),\left(x_{2 k+1}, y_{2 k+1}\right)\right)\right) \\
& \left.+a_{3} D\left(\left(x_{2 k+1}, y_{2 k+1}\right),\left(x_{2 k+1}, y_{2 k+1}\right), \ldots,\left(x_{2 k+1}, y_{2 k+1}\right)\left(x_{2 k+2}, y_{2 k+2}\right)\right)\right) \\
& \left.+a_{4} D\left(\left(x_{2 k}, y_{2 k}\right),\left(x_{2 k}, y_{2 k}\right), \ldots,\left(x_{2 k}, y_{2 k}\right),\left(x_{2 k+2}, y_{2 k+2}\right)\right)\right) \\
= & a_{1} D\left(\left(x_{2 k}, y_{2 k}\right),\left(x_{2 k}, y_{2 k}\right), \ldots,\left(x_{2 k}, y_{2 k}\right),\left(x_{2 k+1}, y_{2 k+1}\right)\right)
\end{aligned}
$$




$$
\begin{aligned}
& \left.+a_{2} D\left(\left(x_{2 k}, y_{2 k}\right),\left(x_{2 k}, y_{2 k}\right), \ldots,\left(x_{2 k}, y_{2 k}\right),\left(x_{2 k+1}, y_{2 k+1}\right)\right)\right) \\
& \left.+a_{3} D\left(\left(x_{2 k+1}, y_{2 k+1}\right),\left(x_{2 k+1}, y_{2 k+1}\right), \ldots,\left(x_{2 k+1}, y_{2 k+1}\right),\left(x_{2 k+2}, y_{2 k+2}\right)\right)\right) \\
& \left.+(n-1) b a_{4}\left[D\left(\left(x_{2 k}, y_{2 k}\right),\left(x_{2 k}, y_{2 k}\right), \ldots,\left(x_{2 k}, y_{2 k}\right),\left(x_{2 k+1}, y_{2 k+1}\right)\right)\right)\right] \\
& \left.+b^{2} a_{4} D\left(\left(x_{2 k+1}, y_{2 k+1}\right),\left(x_{2 k+1}, y_{2 k+1}\right), \ldots,\left(x_{2 k+1}, y_{2 k+1}\right),\left(x_{2 k+2}, y_{2 k+2}\right)\right)\right) \\
& =\left(a_{1}+a_{2}+(n-1) b a_{4}\right) D\left(\left(x_{2 k}, y_{2 k}\right),\left(x_{2 k}, y_{2 k}\right), \ldots,\left(x_{2 k}, y_{2 k}\right),\left(x_{2 k+1}, y_{2 k+1}\right)\right) \\
& \left.\quad \times\left(a_{3}+b^{2} a_{4}\right) D\left(\left(x_{2 k+1}, y_{2 k+1}\right),\left(x_{2 k+1}, y_{2 k+1}\right), \ldots,\left(x_{2 k+1}, y_{2 k+1}\right),\left(x_{2 k+2}, y_{2 k+2}\right)\right)\right) \\
& =\left(a_{1}+a_{2}+(n-1) b a_{4}\right)\left[A\left(x_{2 k}, x_{2 k}, \ldots, x_{2 k}, x_{2 k+1}\right)+A\left(y_{2 k}, y_{2 k}, \ldots, y_{2 k}, y_{2 k+1}\right)\right] \\
& \quad \times\left(a_{3}+b^{2} a_{4}\right)\left[A\left(x_{2 k+1}, x_{2 k+1}, \ldots, x_{2 k+1}, x_{2 k+2}\right)\right. \\
& \left.+A\left(y_{2 k+1}, y_{2 k+1}, \ldots, y_{2 k+1}, y_{2 k+2}\right)\right] .
\end{aligned}
$$

Similarly we obtain

$$
\begin{aligned}
& A\left(y_{2 k+1}, y_{2 k+1}, \ldots, y_{2 k+1}, y_{2 k+2}\right)+A\left(x_{2 k+1}, x_{2 k+1}, \ldots, x_{2 k+1}, x_{2 k+2}\right) \\
& \leqslant\left(a_{1}+a_{2}+(n-1) b a_{4}\right)\left[A\left(y_{2 k}, y_{2 k}, \ldots, y_{2 k}, y_{2 k+1}\right)+A\left(x_{2 k}, x_{2 k}, \ldots, x_{2 k}, x_{2 k+1}\right)\right] \\
& \times\left(a_{3}+b^{2} a_{4}\right)\left[A\left(y_{2 k+1}, y_{2 k+1}, \ldots, y_{2 k+1}, y_{2 k+2}\right)\right. \\
&\left.+A\left(x_{2 k+1}, x_{2 k+1}, \ldots, x_{2 k+1}, x_{2 k+2}\right)\right] .
\end{aligned}
$$

From (2.4) and (2.5), we have

$$
\begin{aligned}
& 2\left[A\left(x_{2 k+1}, x_{2 k+1}, \ldots, x_{2 k+1}, x_{2 k+2}\right)+A\left(y_{2 k+1}, y_{2 k+1}, \ldots, y_{2 k+1}, y_{2 k+2}\right)\right] \\
& \leqslant 2\left[\left(a_{1}+a_{2}+(n-1) b a_{4}\right)\left[A\left(x_{2 k}, x_{2 k}, \ldots, x_{2 k}, x_{2 k+1}\right)+A\left(y_{2 k}, y_{2 k}, \ldots, y_{2 k}, y_{2 k+1}\right)\right]\right] \\
&+2\left[( a _ { 3 } + b ^ { 2 } a _ { 4 } ) \left[A\left(x_{2 k+1}, x_{2 k+1}, \ldots, x_{2 k+1}, x_{2 k+2}\right)\right.\right. \\
&\left.\left.+A\left(y_{2 k+1}, y_{2 k+1}, \ldots, y_{2 k+1}, y_{2 k+2}\right)\right]\right] .
\end{aligned}
$$

This implies that

$$
\begin{gathered}
{\left[A\left(x_{2 k+1}, x_{2 k+1}, \ldots, x_{2 k+1}, x_{2 k+2}\right)+A\left(y_{2 k+1}, y_{2 k+1}, \ldots, y_{2 k+1}, y_{2 k+2}\right)\right]\left[1-\left(a_{3}+b^{2} a_{4}\right)\right]} \\
\leqslant\left[\left(a_{1}+a_{2}+(n-1) b a_{4}\right)\left[A\left(x_{2 k}, x_{2 k}, \ldots, x_{2 k}, x_{2 k+1}\right)+A\left(y_{2 k}, y_{2 k}, \ldots, y_{2 k}, y_{2 k+1}\right)\right]\right]
\end{gathered}
$$

Thus

$$
\begin{aligned}
& A\left(x_{2 k+1}, x_{2 k+1}, \ldots, x_{2 k+1}, x_{2 k+2}\right)+A\left(y_{2 k+1}, y_{2 k+1}, \ldots, y_{2 k+1}, y_{2 k+2}\right) \\
& \quad \leqslant \frac{\left(a_{1}+a_{2}+(n-1) b a_{4}\right)}{1-\left(a_{3}+b^{2} a_{4}\right)}\left[A\left(x_{2 k}, x_{2 k}, \ldots, x_{2 k}, x_{2 k+1}\right)+A\left(y_{2 k}, y_{2 k}, \ldots, y_{2 k}, y_{2 k+1}\right)\right] .
\end{aligned}
$$

Let $\delta=\left[\frac{\left(a_{1}+a_{2}+(n-1) b a_{4}\right)}{1-\left(a_{3}+b^{2} a_{4}\right)}\right]$, then $0 \leqslant \delta<1$ and

$$
\begin{gathered}
A\left(x_{2 k+1}, x_{2 k+1}, \ldots, x_{2 k+1}, x_{2 k+2}\right)+A\left(y_{2 k+1}, y_{2 k+1}, \ldots, y_{2 k+1}, y_{2 k+2}\right) \\
\leqslant \delta\left(A\left(x_{2 k}, x_{2 k}, \ldots, x_{2 k}, x_{2 k+1}\right)+A\left(y_{2 k}, y_{2 k}, \ldots, y_{2 k}, y_{2 k+1}\right)\right) .
\end{gathered}
$$

For all $k \in \mathbb{N}$, applying (2.1) again and by interchanging the roles of $f$ and $g$, we obtain

$$
\begin{aligned}
& A\left(x_{2 k+2}, x_{2 k+2}, \ldots, x_{2 k+2}, x_{2 k+3}\right)+A\left(y_{2 k+2}, y_{2 k+2}, \ldots, y_{2 k+2}, y_{2 k+3}\right) \\
& \quad \leqslant \delta\left(A\left(x_{2 k+1}, x_{2 k+1}, \ldots, x_{2 k+1}, x_{2 k+2}\right)+A\left(y_{2 k+1}, y_{2 k+1}, \ldots, y_{2 k+1}, y_{2 k+2}\right)\right) .
\end{aligned}
$$

It follows from (2.6) that

$$
\begin{aligned}
& A\left(x_{2 k+1}, x_{2 k+1}, \ldots, x_{2 k+1}, x_{2 k+2}\right)+A\left(y_{2 k+1}, y_{2 k+1}, \ldots, y_{2 k+1}, y_{2 k+2}\right) \\
& \leqslant \delta\left(A\left(x_{2 k}, x_{2 k}, \ldots, x_{2 k}, x_{2 k+1}\right)+A\left(y_{2 k}, y_{2 k}, \ldots, y_{2 k}, y_{2 k+1}\right)\right)
\end{aligned}
$$




$$
\begin{aligned}
& \leqslant \delta^{2}\left(A\left(x_{2 k-1}, x_{2 k-1}, \ldots, x_{2 k-1}, x_{2 k}\right)+A\left(y_{2 k-1}, y_{2 k-1}, \ldots, y_{2 k-1}, y_{2 k}\right)\right) \\
& \leqslant \delta^{3}\left(A\left(x_{2 k-2}, x_{2 k-2}, \ldots, x_{2 k-2}, x_{2 k-1}\right)+A\left(y_{2 k-2}, y_{2 k-2}, \ldots, y_{2 k-2}, y_{2 k-1}\right)\right)
\end{aligned}
$$

This implies

$$
\begin{aligned}
& A\left(x_{2 k+1}, x_{2 k+1}, \ldots, x_{2 k+1}, x_{2 k+2}\right)+A\left(y_{2 k+1}, y_{2 k+1}, \ldots, y_{2 k+1}, y_{2 k+2}\right) \\
& \quad \leqslant \delta^{2 k+1}\left(A\left(x_{0}, x_{0}, \ldots, x_{0}, x_{1}\right)+A\left(y_{0}, y_{0}, \ldots, y_{0}, y_{1}\right)\right) .
\end{aligned}
$$

Similarly, by (2.7), we get

$$
\begin{aligned}
& A\left(x_{2 k+2}, x_{2 k+2}, \ldots, x_{2 k+2}, x_{2 k+3}\right)+A\left(y_{2 k+2}, y_{2 k+2}, \ldots, y_{2 k+2}, y_{2 k+3}\right) \\
& \quad \leqslant \delta^{2 k+2}\left(A\left(x_{0}, x_{0}, \ldots, x_{0}, x_{1}\right)+A\left(y_{0}, y_{0}, \ldots, y_{0}, y_{1}\right)\right) .
\end{aligned}
$$

By Lemma 1.11, we have for all $k, m \in \mathbb{N}$ with $k \leqslant m$

$$
\begin{aligned}
A\left(x_{2 k+1}, x_{2 k+1}, \ldots, x_{2 k+1}, x_{2 m+1}\right) \leqslant & b(n-1) A\left(x_{2 k+1}, x_{2 k+1}, \ldots, x_{2 k+1}, x_{2 k+2}\right) \\
& +b^{2} A\left(x_{2 k+2}, x_{2 k+2}, \ldots, x_{2 k+2}, x_{2 m+1}\right)
\end{aligned}
$$

and

$$
\begin{aligned}
A\left(y_{2 k+1}, y_{2 k+1}, \ldots, y_{2 k+1}, y_{2 m+1}\right) \leqslant & b(n-1) A\left(y_{2 k+1}, y_{2 k+1}, \ldots, y_{2 k+1}, y_{2 k+2}\right) \\
& +b^{2} A\left(y_{2 k+2}, y_{2 k+2}, \ldots, y_{2 k+2}, y_{2 m+1}\right) .
\end{aligned}
$$

So we have

$$
\begin{aligned}
A( & \left.x_{2 k+1}, x_{2 k+1}, \ldots, x_{2 k+1}, x_{2 m+1}\right)+A\left(y_{2 k+1}, y_{2 k+1}, \ldots, y_{2 k+1}, y_{2 m+1}\right) \\
\leqslant & b\left[(n-1)\left(A\left(x_{2 k+1}, x_{2 k+1}, \ldots, x_{2 k+1}, x_{2 k+2}\right)+(n-1) A\left(y_{2 k+1}, y_{2 k+1}, \ldots, y_{2 k+1}, y_{2 k+2}\right)\right)\right] \\
& +b^{2}\left[A\left(x_{2 k+2}, x_{2 k+2}, \ldots, x_{2 k+2}, x_{2 m+1}\right)+A\left(y_{2 k+2}, y_{2 k+2}, \ldots, y_{2 k+2}, y_{2 m+1}\right)\right] \\
= & b(n-1)\left[A\left(x_{2 k+1}, x_{2 k+1}, \ldots, x_{2 k+1}, x_{2 k+2}\right)+A\left(y_{2 k+1}, y_{2 k+1}, \ldots, y_{2 k+1}, y_{2 k+2}\right)\right] \\
& +b^{3}(n-1)\left[A\left(x_{2 k+2}, x_{2 k+2}, \ldots, x_{2 k+2}, x_{2 k+3}\right)+A\left(y_{2 k+2}, y_{2 k+2}, \ldots, y_{2 k+2}, y_{2 k+3}\right)\right] \\
& \vdots \\
& +b^{2(2 m-2 k)-1}(n-1)\left[A\left(x_{2 m-1}, x_{2 m-1}, \ldots, x_{2 m-1}, x_{2 m}\right)+A\left(y_{2 m-1}, y_{2 m-1}, \ldots, y_{2 m-1}, y_{2 m}\right)\right] \\
& +b^{2(2 m-2 k)}(n-1)\left[A\left(x_{2 m}, x_{2 m}, \ldots, x_{2 m}, x_{2 m+1}\right)+A\left(y_{2 m}, y_{2 m}, \ldots, y_{2 m}, y_{2 m+1}\right)\right] \\
\Rightarrow & A\left(x_{2 k+1}, x_{2 k+1}, \ldots, x_{2 k+1}, x_{2 m+1}\right)+A\left(y_{2 k+1}, y_{2 k+1}, \ldots, y_{2 k+1}, y_{2 m+1}\right) \\
\leqslant & b(n-1)\left[A\left(x_{2 k+1}, x_{2 k+1}, \ldots, x_{2 k+1}, x_{2 k+2}\right)+A\left(y_{2 k+1}, y_{2 k+1}, \ldots, y_{2 k+1}, y_{2 k+2}\right)\right] \\
& +b^{3}(n-1)\left[A\left(x_{2 k+2}, x_{2 k+2}, \ldots, x_{2 k+2}, x_{2 k+3}\right)+A\left(y_{2 k+2}, y_{2 k+2}, \ldots, y_{2 k+2}, y_{2 k+3}\right)\right] \\
& \vdots \\
& +b^{2(2 m-2 k)}(n-1)\left[A\left(x_{2 m}, x_{2 m}, \ldots, x_{2 m}, x_{2 m+1}\right)+A\left(y_{2 m}, y_{2 m}, \ldots, y_{2 m}, y_{2 m+1}\right)\right] \\
= & b(n-1)\left[\delta^{2 k+1}+b^{2} \delta^{2 k+2}+\ldots+b^{2(2 m-2 k)-1} \delta^{2 m}\right] \times\left[A\left(x_{0}, x_{0}, \ldots, x_{0}, x_{1}\right)+A\left(y_{0}, y_{0}, \ldots, y_{0}, y_{1}\right)\right] \\
\Rightarrow & A\left(x_{2 k+1}, x_{2 k+1}, \ldots, x_{2 k+1}, x_{2 m+1}\right)+A\left(y_{2 k+1}, y_{2 k+1}, \ldots, y_{2 k+1}, y_{2 m+1}\right) \\
\leqslant & b(n-1) \frac{\delta^{2 k+1}}{1-\delta b^{2}}\left[\left(A\left(x_{0}, x_{0}, \ldots, x_{0}, x_{1}\right)+A\left(y_{0}, y_{0}, \ldots, y_{0}, y_{1}\right)\right)\right] .
\end{aligned}
$$

Similarly, we have

$$
A\left(x_{2 k}, x_{2 k}, \ldots, x_{2 k}, x_{2 m+1}\right)+A\left(y_{2 k}, y_{2 k}, \ldots, y_{2 k}, y_{2 m+1}\right)
$$




$$
\leqslant(n-1) \frac{b \delta^{2 k}}{1-\delta b^{2}}\left(A\left(x_{0}, x_{0}, \ldots, x_{0}, x_{1}\right)+A\left(y_{0}, y_{0}, \ldots, y_{0}, y_{1}\right)\right)
$$

and

$$
\begin{aligned}
& A\left(x_{2 k}, x_{2 k}, \ldots, x_{2 k}, x_{2 m}\right)+A\left(y_{2 k}, y_{2 k}, \ldots, y_{2 k}, y_{2 m}\right) \\
& \quad=(n-1) \frac{b \delta^{2 k}}{1-\delta b^{2}}\left(A\left(x_{0}, x_{0}, \ldots, x_{0}, x_{1}\right)+A\left(y_{0}, y_{0}, \ldots, y_{0}, y_{1}\right)\right) .
\end{aligned}
$$

Hence, for all $k, m \in \mathbb{N}$ with $k \leqslant m$, we have

$$
A\left(x_{k}, x_{k}, \ldots, x_{k}, x_{m}\right)+A\left(y_{k}, y_{k}, \ldots, y_{k}, y_{m}\right) \leqslant(n-1) \frac{b \delta^{k}}{1-\delta b^{2}}\left(A\left(x_{0}, x_{0}, \ldots, x_{0}, x_{1}\right)+A\left(y_{0}, y_{0}, \ldots, y_{0}, y_{1}\right)\right)
$$

Since $0 \leqslant \delta=\left[\frac{\left(\alpha_{1}+\alpha_{2}+(n-1) b \alpha_{4}\right)}{1-\left(\alpha_{3}+b^{2} \alpha_{4}\right)}\right]<1$, we have

$$
\lim _{k, m \rightarrow \infty}\left(A\left(x_{k}, x_{k}, \ldots, x_{k}, x_{m}\right)+A\left(y_{k}, y_{k}, \ldots, y_{k}, y_{m}\right)\right)=0
$$

That is,

$$
\lim _{k, m \rightarrow \infty} A\left(x_{k}, x_{k}, \ldots, x_{k}, x_{m}\right)=\lim _{k, m \rightarrow \infty} A\left(y_{k}, y_{k}, \ldots, y_{k}, y_{m}\right)=0
$$

Therefore, $\left\{x_{k}\right\}$ and $\left\{y_{k}\right\}$ are both Cauchy sequences in $X$. By the completeness of $X$, there exist $x, y \in X$ such that $x_{k} \rightarrow x$ and $y_{k} \rightarrow y$ as $k \rightarrow \infty$.

We next show that the pair $(x, y)$ is a coupled common fixed point of $f$ and $g$.

Now, suppose $f$ is continuous, then we have

$$
x=\lim _{k \rightarrow \infty} x_{2 k+1}=\lim _{k \rightarrow \infty} f\left(x_{2 k}, y_{2 k}\right)=f\left(\lim _{k \rightarrow \infty} x_{2 k}, \lim _{k \rightarrow \infty} y_{2 k}\right)=f(x, y)
$$

and

$$
y=\lim _{k \rightarrow \infty} y_{2 k+1}=\lim _{k \rightarrow \infty} f\left(y_{2 k}, x_{2 k}\right)=f\left(\lim _{k \rightarrow \infty} y_{2 k}, \lim _{k \rightarrow \infty} x_{2 k}\right)=f(y, x)
$$

Applying (2.1), we have

$$
\begin{aligned}
A( & f(x, y), f(x, y), \ldots, f(x, y), g(x, y))+A(f(y, x), f(y, x), \ldots, f(y, x), g(y, x)) \\
\leqslant & a_{1} D((x, y),(x, y), \ldots,(x, y),(x, y)) \\
& +a_{2} D((x, y),(x, y), \ldots,(x, y),(f(x, y), f(y, x)))+a_{3} D((x, y),(x, y), \ldots,(x, y),(g(x, y), g(y, x))) \\
& +a_{4} D((x, y),(x, y), \ldots,(x, y),(g(x, y), g(y, x)))+a_{5} D((x, y),(x, y), \ldots,(x, y),(f(x, y), f(y, x))) \\
= & a_{2} D((x, y),(x, y), \ldots,(x, y),(x, y))+a_{3} D((x, y),(x, y), \ldots,(x, y),(g(x, y), g(y, x))) \\
& +a_{4} D((x, y),(x, y), \ldots,(x, y),(g(x, y), g(y, x)))+a_{5} D((x, y),(x, y), \ldots,(x, y),(x, y)) \\
= & \left(a_{3}+a_{4}\right) D((x, y),(x, y), \ldots,(x, y),(g(x, y), g(y, x))) \\
= & \left(a_{3}+a_{4}\right)(A(x, x, \ldots, x, g(x, y))+(A(y, y, \ldots, y, g(y, x))) .
\end{aligned}
$$

Therefore

$$
A(x, x, \ldots, x, g(x, y))+A(y, y, \ldots, y, g(y, x)) \leqslant\left(a_{3}+a_{4}\right)(A(x, x, \ldots, x, g(x, y))+A(y, y, \ldots, y, g(y, x))) .
$$

Since $0 \leqslant\left(a_{3}+a_{4}\right)<1,(A(x, x, x, \ldots, x, g(x, y))=A(y, y, y, \ldots, y, g(y, x))=0$. That is, $g(x, y)=x$ and $g(y, x)=y$. This implies $(x, y)$ is a coupled fixed point of $g$.

In a similar fashion, suppose $\mathrm{g}$ is continuous, then we have

$$
x=\lim _{k \rightarrow \infty} x_{2 k+2}=\lim _{k \rightarrow \infty} g\left(x_{2 k+1}, y_{2 k+1}\right)=g\left(\lim _{k \rightarrow \infty} x_{2 k+1}, \lim _{k \rightarrow \infty} y_{2 k+1}\right)=g(x, y)
$$


and

$$
y=\lim _{k \rightarrow \infty} y_{2 k+2}=\lim _{k \rightarrow \infty} g\left(y_{2 k+1}, x_{2 k+1}\right)=g\left(\lim _{k \rightarrow \infty} y_{2 k+1}, \lim _{k \rightarrow \infty} x_{2 k+1}\right)=g(y, x) .
$$

Applying (2.1) again, we also get

$$
A(x, x, \ldots, x, f(x, y))+A(y, y, \ldots, y, f(y, x)) \leqslant\left(a_{2}+a_{5}\right)(A(x, x, \ldots, x, f(x, y))+A(x, x, \ldots, x, f(y, x))) .
$$

This implies $f(x, y)=x$ and $f(y, x)=y$ and so $(x, y)$ is as well a coupled fixed point of $f$. Therefore, $(x, y)$ is a coupled common fixed point of $f$ and $g$.

Finally, suppose $X$ satisfies hypotheses 3 (a) and 3 (b). Then by (2.3) we get $x_{k} \preceq x$ and $y \preceq y_{k}$ for all $\mathrm{k} \in \mathbb{N}$. Applying Lemmas 1.11 and 1.12, we obtain

$$
\begin{aligned}
& D((x, y),(x, y), \ldots,(f(x, y), f(y, x))) \\
& \leqslant b(n-1) D\left((x, y),(x, y), \ldots,(x, y),\left(x_{2 k+2}, y_{2 k+2}\right)\right) \\
&+b^{2} D\left(\left(x_{2 k+2}, y_{2 k+2}\right),\left(x_{2 k+2}, y_{2 k+2}\right), \ldots,\left(x_{2 k+2}, y_{2 k+2}\right),(f(x, y), f(y, x))\right) \\
&= b(n-1) D\left((x, y),(x, y), \ldots,(x, y),\left(x_{2 k+2}, y_{2 k+2}\right)\right) \\
&+b^{2} D\left(g\left(x_{2 k+1}, y_{2 k+1}\right), g\left(y_{2 k+1}, x_{2 k+1}\right)\right),\left(g\left(x_{2 k+1}, y_{2 k+1}\right), g\left(y_{2 k+1}, x_{2 k+1}\right)\right), \ldots \\
& \ldots,\left(g\left(x_{2 k+1}, y_{2 k+1}\right), g\left(y_{2 k+1}, x_{2 k+1}\right)\right),(f(x, y), f(y, x)) \\
& \leqslant b(n-1) D\left((x, y),(x, y), \ldots,(x, y),\left(x_{2 k+2}, y_{2 k+2}\right)\right) \\
&+b^{2} A\left(g\left(x_{2 k+1}, y_{2 k+1}\right), g\left(x_{2 k+1}, y_{2 k+1}\right), \ldots, g\left(x_{2 k+1}, y_{2 k+1}\right), f(x, y)\right) \\
&+b^{2} A\left(g\left(y_{2 k+1}, x_{2 k+1}\right), g\left(y_{2 k+1}, x_{2 k+1}\right), \ldots, g\left(y_{2 k+1}, x_{2 k+1}\right), f(y, x)\right) .
\end{aligned}
$$

This implies

$$
\begin{aligned}
& D((x, y),(x, y), \ldots,(f(x, y), f(y, x))) \\
& \leqslant\left.b(n-1) A\left(x, x, \ldots, x, x_{2 k+2}\right)+b(n-1) A\left(y, y, \ldots, y, y_{2 k+2}\right)\right) \\
&+b^{2} A\left(g\left(x_{2 k+1}, y_{2 k+1}\right), g\left(x_{2 k+1}, y_{2 k+1}\right), \ldots, g\left(x_{2 k+1}, y_{2 k+1}\right), f(x, y)\right) \\
&+b^{2} A\left(g\left(y_{2 k+1}, x_{2 k+1}\right), g\left(y_{2 k+1}, x_{2 k+1}\right), \ldots, g\left(y_{2 k+1}, x_{2 k+1}\right), f(y, x)\right) .
\end{aligned}
$$

By using (2.1) and interchanging the roles of $f$ with $g$ we obtain

$$
\begin{aligned}
A(g & \left.\left(x_{2 k+1}, y_{2 k+1}\right), g\left(x_{2 k+1}, y_{2 k+1}\right), \ldots, g\left(x_{2 k+1}, y_{2 k+1}\right), f(x, y)\right) \\
& +A\left(g\left(y_{2 k+1}, x_{2 k+1}\right), g\left(y_{2 k+1}, x_{2 k+1}\right), \ldots, g\left(y_{2 k+1}, x_{2 k+1}\right), f(y, x)\right) \\
\leqslant & a_{1} D\left(\left(x_{2 k+1}, y_{2 k+1}\right),\left(x_{2 k+1}, y_{2 k+1}\right) \ldots,\left(x_{2 k+1}, y_{2 k+1}\right),(x, y)\right) \\
& +a_{2} D\left(\left(x_{2 k+1}, y_{2 k+1}\right),\left(x_{2 k+1}, y_{2 k+1}\right) \ldots,\left(x_{2 k+1}, y_{2 k+1}\right),\left(g\left(x_{2 k+1}, y_{2 k+1}\right), g\left(y_{2 k+1}, x_{2 k+1}\right)\right)\right) \\
& +a_{3} D((x, y),(x, y), \ldots,(x, y),(f(x, y), f(y, x))) \\
& +a_{4} D\left(\left(x_{2 k+1}, y_{2 k+1}\right),\left(x_{2 k+1}, y_{2 k+1}\right), \ldots,\left(x_{2 k+1}, y_{2 k+1}\right),(f(x, y), f(y, x))\right) \\
& +a_{5} D\left((x, y),(x, y), \ldots,(x, y),\left(g\left(x_{2 k+1}, y_{2 k+1}\right), g\left(y_{2 k+1}, x_{2 k+1}\right)\right)\right) \\
= & a_{1} D\left(\left(x_{2 k+1}, y_{2 k+1}\right),\left(x_{2 k+1}, y_{2 k+1}\right) \ldots,\left(x_{2 k+1}, y_{2 k+1}\right),(x, y)\right) \\
& +a_{2} D\left(\left(x_{2 k+1}, y_{2 k+1}\right),\left(x_{2 k+1}, y_{2 k+1}\right) \ldots,\left(x_{2 k+1}, y_{2 k+1}\right),\left(x_{2 k+2}, y_{2 k+2}\right)\right) \\
& +a_{3} D((x, y),(x, y), \ldots,(x, y),(f(x, y), f(y, x))) \\
& +a_{4} D\left(\left(x_{2 k+1}, y_{2 k+1}\right),\left(x_{2 k+1}, y_{2 k+1}\right), \ldots,\left(x_{2 k+1}, y_{2 k+1}\right),(f(x, y), f(y, x))\right) \\
& +a_{5} D\left((x, y),(x, y), \ldots,(x, y),\left(x_{2 k+2}, y_{2 k+2}\right)\right) .
\end{aligned}
$$

It follows from (2.8) and (2.9) that

$$
D((x, y),(x, y), \ldots,(f(x, y), f(y, x)))
$$




$$
\begin{aligned}
\leqslant & b\left[(n-1) A\left(x, x, \ldots, x, x_{2 k+2}\right)+(n-1) A\left(y, y, \ldots, y, y_{2 k+2}\right)\right] \\
& +b^{2}\left[a_{1} D\left(\left(x_{2 k+1}, y_{2 k+1}\right),\left(x_{2 k+1}, y_{2 k+1}\right) \ldots,\left(x_{2 k+1}, y_{2 k+1}\right),(x, y)\right)\right. \\
& +a_{2} D\left(\left(x_{2 k+1}, y_{2 k+1}\right),\left(x_{2 k+1}, y_{2 k+1}\right), \ldots,\left(x_{2 k+1}, y_{2 k+1}\right),\left(x_{2 k+2}, y_{2 k+2}\right)\right) \\
& +a_{3} D((x, y),(x, y), \ldots,(x, y),(f(x, y), f(y, x))) \\
& +a_{4} D\left(\left(x_{2 k+1}, y_{2 k+1}\right),\left(x_{2 k+1}, y_{2 k+1}\right), \ldots,\left(x_{2 k+1}, y_{2 k+1}\right),(f(x, y), f(y, x))\right) \\
& \left.+a_{5} D\left((x, y),(x, y), \ldots,(x, y),\left(x_{2 k+2}, y_{2 k+2}\right)\right)\right] .
\end{aligned}
$$

Taking the limit as $k \rightarrow \infty$ in (2.10), we get

$$
\begin{aligned}
& D((x, y),(x, y), \ldots,(f(x, y), f(y, x))) \\
& \leqslant b[(n-1) A(x, x, \ldots, x, x)+(n-1) A(y, y, \ldots, y, y)] b^{2}\left[a_{1} D((x, y),(x, y), \ldots,(x, y),(x, y))\right. \\
& \quad+a_{2} D((x, y),(x, y) \ldots,(x, y),(x, y))+a_{3} D((x, y),(x, y), \ldots,(x, y),(f(x, y), f(y, x))) \\
& \left.\quad+a_{4} D((x, y),(x, y), \ldots,(x, y),(f(x, y), f(y, x)))+a_{5} D((x, y),(x, y), \ldots,(x, y),(x, y))\right] \\
& =b^{2}\left[a_{3} D((x, y),(x, y), \ldots,(x, y),(f(x, y), f(y, x)))+a_{4} D((x, y),(x, y), \ldots,(x, y),(f(x, y), f(y, x)))\right] .
\end{aligned}
$$

Therefore,

$$
D((x, y),(x, y), \ldots,(f(x, y), f(y, x))) \leqslant b^{2}\left(a_{3}+a_{4}\right) D((x, y),(x, y), \ldots,(x, y),(f(x, y), f(y, x))) .
$$

Since $b^{2}\left(a_{3}+a_{4}\right)<1$, we have $D((x, y),(x, y), \ldots,(x, y),(f(x, y), f(y, x)))=0$. That is $f(x, y)=x$ and $f(y, x)=y$. This implies $(x, y)$ is a coupled fixed point of $f$.

Similarly, we can show that $g(x, y)=x$ and $g(y, x)=y$.

Hence, $f(x, y)=x=g(x, y)$ and $f(y, x)=y=g(y, x)$. Thus $(x, y)$ is a coupled common fixed point of $f$ and $g$. This completes the proof.

Theorem 2.2. In addition to the hypotheses of Theorem 2.1, if $\mathrm{X}$ is a totally ordered set, then $\mathrm{f}$ and $\mathrm{g}$ have a unique coupled common fixed point. Furthermore, any fixed point of $\mathrm{f}$ is a fixed point of $\mathrm{g}$, and conversely.

Proof. Let $X$ be a totally ordered set. Suppose $(x, y),\left(x^{*}, y^{*}\right)$ are coupled common fixed points of $f$ and g. That is, $f(x, y)=x, f(y, x)=y$, and $g\left(x^{*}, y^{*}\right)=x^{*}, g\left(y^{*}, x^{*}\right)=y^{*}$. We show that $x=x^{*}, y=y^{*}$ and subsequently $x=y$.

Observe that if $X$ is a totally ordered set, then, for every $(x, y),\left(x^{*}, y^{*}\right) \in X \times X$, there exists $(u, v) \in$ $X \times X$ that is comparable to $(x, y)$ and $\left(x^{*}, y^{*}\right)$.

So we let $(x, y) \preceq\left(x^{*}, y^{*}\right)$ without loss of generality, then it follows from Lemma 1.12 and Theorem 2.1 that

$$
\begin{aligned}
D & \left((x, y),(x, y), \ldots,(x, y),\left(x^{*}, y^{*}\right)\right) \\
= & A\left(x, x, \ldots, x, x^{*}\right)+A\left(y, y, \ldots, y, y^{*}\right) \\
= & A\left(f(x, y), f(x, y), \ldots, f(x, y), g\left(x^{*}, y^{*}\right)\right)+A\left(f(y, x), f(y, x), \ldots, f(y, x), g\left(y^{*}, x^{*}\right)\right) \\
\leqslant & a_{1} D\left((x, y),(x, y), \ldots,(x, y),\left(x^{*}, y^{*}\right)\right)+a_{2} D((x, y),(x, y) \ldots,(x, y),(f(x, y), f(y, x)) \\
& +a_{3} D\left(\left(x^{*}, y^{*}\right),\left(x^{*}, y^{*}\right), \ldots,\left(x^{*}, y^{*}\right),\left(g\left(x^{*}, y^{*}\right), g\left(y^{*}, x^{*}\right)\right)\right) \\
& +a_{4} D\left((x, y),(x, y), \ldots,(x, y),\left(g\left(x^{*}, y^{*}\right), g\left(y^{*}, x^{*}\right)\right)\right)+a_{5} D\left(\left(x^{*}, y^{*}\right),\left(x^{*}, y^{*}\right), \ldots,\left(x^{*}, y^{*}\right),(f(x, y), f(y, x))\right) \\
= & a_{1} D\left((x, y),(x, y), \ldots,(x, y),\left(x^{*}, y^{*}\right)\right)+a_{2} D((x, y),(x, y) \ldots,(x, y),(x, y)) \\
& +a_{3} D\left(\left(x^{*}, y^{*}\right),\left(x^{*}, y^{*}\right), \ldots,\left(x^{*}, y^{*}\right),\left(x^{*}, y^{*}\right)\right) \\
& +a_{4} D\left((x, y),(x, y), \ldots,(x, y),\left(x^{*}, y^{*}\right)\right)+a_{5} D\left(\left(x^{*}, y^{*}\right),\left(x^{*}, y^{*}\right), \ldots,\left(x^{*}, y^{*}\right),(x, y)\right) \\
= & a_{1} D\left((x, y),(x, y), \ldots,(x, y),\left(x^{*}, y^{*}\right)\right)+a_{4} D\left((x, y),(x, y), \ldots,(x, y),\left(x^{*}, y^{*}\right)\right) \\
& +a_{5} D\left(\left(x^{*}, y^{*}\right),\left(x^{*}, y^{*}\right), \ldots,\left(x^{*}, y^{*}\right),(x, y)\right) \\
= & \left(a_{1}+a_{4}+a_{5}\right) D\left((x, y),(x, y), \ldots,(x, y),\left(x^{*}, y^{*}\right)\right) .
\end{aligned}
$$


Since $0 \leqslant\left(a_{1}+b^{2} a_{4}+a_{5}\right)<1$, we have $D\left((x, y),(x, y), \ldots,(x, y),\left(x^{*}, y^{*}\right)\right)=0$, which implies $x=x^{*}$ and $y=y^{*}$.

Proceeding, we show that any fixed point of $f$ is a fixed point of $g$, and conversely. Applying Lemma 1.12 and Theorem 2.1 we have

$$
\begin{aligned}
A & (x, x, \ldots, x, y)+A(y, y, \ldots, y, x) \\
= & A(f(x, y), f(x, y), \ldots, f(x, y), g(y, x))+A(f(y, x), f(y, x), \ldots, f(y, x), g(x, y)) \\
\leqslant & a_{1} D((x, y),(x, y), \ldots,(x, y),(y, x))+a_{2} D((x, y),(x, y) \ldots,(x, y),(f(x, y), f(y, x)) \\
& +a_{3} D((y, x),(y, x), \ldots,(y, x),(g(y, x), g(x, y))) \\
& +a_{4} D((x, y),(x, y), \ldots,(x, y),(g(y, x), g(x, y)))+a_{5} D((y, x),(y, x), \ldots,(y, x),(f(x, y), f(y, x))) \\
= & a_{1} D((x, y),(x, y), \ldots,(x, y),(y, x))+a_{2} D((x, y),(x, y) \ldots,(x, y),(x, y)) \\
& +a_{3} D((y, x),(y, x), \ldots,(y, x),(y, x)) \\
& +a_{4} D((x, y),(x, y), \ldots,(x, y),(y, x))+a_{5} D((y, x),(y, x), \ldots,(y, x),(x, y)) \\
= & a_{1} D((x, y),(x, y), \ldots,(x, y),(y, x))+a_{4} D((x, y),(x, y), \ldots,(x, y),(y, x)) \\
& +a_{5} D((x, y),(x, y), \ldots,(x, y),(y, x)) .
\end{aligned}
$$

Therefore

$$
A(x, x, \ldots, x, y)+A(y, y, \ldots, y, x) \leqslant\left(a_{1}+a_{4}+a_{5}\right)(A(x, x, \ldots, x, y)+A(y, y, \ldots, y, x)) .
$$

Since $\left(a_{1}+b^{4} a_{4}+a_{5}\right)<1, A(x, x, \ldots, x, y)+A(y, y, \ldots, y, x)=0$. That is, $x=y$. The coupled common fixed point of $f$ and $g$ is unique.

Example 2.3. Let $(\mathbb{R}, \preceq, A)$ be totally ordered complete $A_{b}$-metric space with $A_{b}$-metric defined as in Example 1.7. Let $f, g: \mathbb{R} \rightarrow \mathbb{R}$ be two maps defined by $f(x, y)=\frac{6 x-3 y+24 n-3}{24 n}$ and $g(x, y)=\frac{8 x-4 y+32 n-4}{32 n}$ for all $n \geqslant 1$. The pair $(f, g)$ has the mixed weakly monotone property on $R$ and

$$
\begin{aligned}
& A(f(x, y), f(x, y), \ldots, f(x, y), g(u, v))+A(f(y, x), f(y, x), \ldots, f(y, x), g(v, u)) \\
& =(n-1)|f(x, y)-g(u, v)|+(n-1)|f(y, x)-g(v, u)| \\
& =(n-1)\left|\frac{6 x-3 y+24 n-3}{24 n}-\frac{8 u-4 v+32 n-4}{32 n}\right|+(n-1)\left|\frac{6 y-3 x+24 n-3}{24 n}-\frac{8 v-4 u+32 n-4}{32 n}\right| \\
& =\frac{(n-1)}{768 n}|192(x-u)+96(y-v)|+\frac{(n-1)}{768 n}|192(y-v)+96(x-u)| \\
& \leqslant \frac{192(n-1)}{768 n}(|| x-u|+| y-v|+| y-v|+| x-u||) \\
& =\frac{(n-1)}{2 n}(|| x-u|+| y-v||) .
\end{aligned}
$$

Then the contractive condition (2.2) is satisfied with $a_{1}=\frac{n-1}{n}, a_{2}=a_{3}=a_{4}=a_{5}=0$. Moreover, (1.1) is the unique coupled common fixed point of $f$ and $g$.

\section{References}

[1] M. Abbas, B. Ali, Y. I Suleiman, Generalized coupled common fixed point results in partially ordered A-metric spaces, Fixed point theory Appl., 2015 (2015), 24 pages. 1, 1.1, 1, 1.2, 1.3, 1

[2] M. Abbas, M. Ali Khan, S. Radenović, Common coupled fixed point theorems in cone metric spaces for $w$-compatible mappings, Appl. Math. Comput., 217 (2010), 195-202. 1

[3] T. Abeljawad, K. Abodayeh, N. M. Mlaiki, On fixed point generalizations to partial b-metric spaces, J. Comput. Anal. Appl., 19 (2015), 883-891.

[4] A. Aghajani, M. Abbas, E. Pourhadi Kallehbasti, Coupled fixed point theorems in partially ordered metric spaces and application, Math. Commun., 17 (2012), 497-509. 
[5] I. A. Bakhtin, The contraction mapping principle in almost metric space, (Russian) Functional analysis, Ulyanovsk. Gos. Ped. Inst., Ulyanovsk, (1989), 26-37. 1, 1, 1, 1

[6] T. Gnana Bhaskar, V. Lakshmikantham, Fixed point theorems in partially ordered metric spaces and applications, Nonlinear Anal., 65 (2006), 1379-1393. 1.4, 1

[7] D. J. Guo, V. Lakshmikantham, Coupled fixed points of nonlinear operators with applications, Nonlinear Anal., 11 (1987), 623-632. 1

[8] Z. Kadelburg, P. Kumam, S. Radenović, W. Sintunavarat, Common coupled fixed point theorems for Geraghty-type contraction mappings using monotone property, Fixed Point Theory Appl., 2015 (2015), 14 pages. 1

[9] Z. Kadelburg, S. Radenović, Coupled fixed point results under tvs-cone metric and w-cone-distance, Adv. Fixed point Theory, 2 (2012), 29-46. 1

[10] E. Karapinar, Coupled Fixed Point on Cone Metric Spaces, Gazi Univ. J. Sci., 1 (2011) 51-58.

[11] E. Karapınar, D. T'urkoğlu, Best approximations theorem for a couple in cone Banach space, Fixed Point Theory Appl., 2010 (2010), 9 pages. 1

[12] B. Khomdram, Y. Rohen, T. C. Singh, Coupled fixed point theorems in $\mathrm{G}_{\mathrm{b}}$-metric space satisfying some rational contractive conditions, SpringerPlus, 5 (2016), 1-16. 1

[13] W. Long, B. E. Rhoades, M. Rajović, Coupled coincidence points for two mappings in metric spaces and cone metric spaces, Fixed Point Theory Appl., 2012 (2012), 9 pages. 1

[14] J. G. Mehta, M. L. Joshi,On coupled fixed point theorem in partially ordered complete metric space, Int. J. Pure Appl. Sci. Technol., 1 (2010), 87-92. 1

[15] N. Mlaiki, A. Zarrad, N. Souayah, A. Mukheimer, T. Abdeljawed, Fixed point theorems in $\mathrm{M}_{\mathrm{b}}$-metric spaces, J. Math. Anal., 7 (2016), 1-9. 1

[16] V. Ozturk, S. Radenović, Some remarks on b-(E.A)-property in b-metric spaces, SpringerPlus, 5 (2016), 1-10.

[17] S. Radenović, A note on tripled coincidence and tripled common fixed point theorems in partially ordered metric spaces, Appl. Math. Comput., 236 (2014), 367-372.

[18] S. Radenović, Coupled fixed point theorems for monotone mappings in partially ordered metric spaces, Kragujevac J. Math., 38 (2014), 249-257.

[19] S. Radenović, Remarks on some coupled coincidence point results in partially ordered metric spaces, Arab J. Math. Sci., 20 (2014), 29-39. 1

[20] W. Shantanawi, Some common coupled fixed point results in cone metric spaces, Int. J. Math. Anal. (Ruse), 4 (2010), 2381-2388. 1

[21] N. Souayah, A fixed point in partial $S_{\mathrm{b}}$-metric spaces, An. Şt. Univ. Ovidius Constanta, 24 (2016), 351-362. 1

[22] N. Souayah, N. Mlaiki, A fixed point theorem in $\mathrm{S}_{\mathrm{b}}$-metric spaces, J. Math. Computer Sci., 16 (2016), 131-139.

[23] M. Ughade, D. Turkoglu, S. R. Singh, R. D. Daheriya, Some fixed point theorems in $A_{b}-$ metric space, British J. Math. Comput. Sci., 19 (2016), 1-24. 1, 1, 1.5, 1.7, 1.8, 1.10 